\title{
Satisfação e perfil de usuários do serviço odontológico no Sistema Único de Saúde
}

\author{
Satisfaction and profile of dental care users in the Unified Health System
}

\author{
Suzely Adas Saliba Moimaz \\ Denise de Toledo Rós** \\ Danielle Bordin ${ }^{* * *}$ \\ Tania Adas Saliba Rovida*** \\ Cléa Adas Saliba Garbin ${ }^{* * * * *}$
}

\section{Resumo}

Introdução: uma das formas de fomentar a qualidade e a resolubilidade do sistema de saúde é com a avaliação do serviço de saúde prestado, entretanto, em função da complexidade envolvida, essa atitude é ainda um desafio na maioria dos serviços. Objetivo: o objetivo desta pesquisa é avaliar a satisfação do usuário com o serviço público odontológico, de acordo com suas características socioeconômicas e demográficas e com a resolubilidade do serviço prestado. Materiais e método: trata-se de uma pesquisa transversal do tipo inquérito, cuja população de estudo foi formada por usuários do serviço odontológico do Sistema Único de Saúde. O cálculo da amostra foi realizado considerando a população adulta estimada, com uma precisão de 5\%, intervalo de confiança de $95 \%$ e efeito de desenho 1, para uma prevalência de $50 \%$ de adultos satisfeitos, totalizando 461 usuários. A técnica utilizada para coleta dos dados foi a entrevista interpessoal padronizada, realizadas nas unidades de saúde da família, empregando-se um formulário estruturado. Resultados: a amostra teve predomínio de mulheres $(70,8 \%)$, adultos jovens $(71,0 \%)$, solteiros $(57,9 \%)$, baixa renda $(49,4 \%)$, baixa escolaridade (50,3\%). A maioria dos usuários estava satisfeita com o serviço prestado (76,2\%), e a satisfação não teve associação estatística com gênero, idade, estado civil, escolaridade e ocupação ( $p>0,05)$, entretanto, quanto à renda do usuário e à resolubilidade do serviço, a associação foi significativa $(p<0,05)$. Conclusão: os usuários entrevistados demonstraram satisfação elevada com os serviços odontológicos, apenas a renda e a resolubilidade configuraram-se como fatores incisivos na satisfação dos usuários.

Palavras-chave: Avaliação em saúde. Atenção primária à saúde. Saúde bucal.

\section{Introdução}

No Brasil, o sistema de saúde vigente prioriza a Atenção Primária em Saúde (APS) ${ }^{1}$, que é um serviço que oferece a entrada no sistema de todas as necessidades e problemas do usuário e oferece atenção integral ao indivíduo em todas as fases da vida, tratando os problemas mais comuns do núcleo familiar e da comunidade. Em casos em que o atendimento secundário ou terciário são necessários, o

Professora titular, Departamento de Odontologia Preventiva e Social, Faculdade de Odontologia de Araçatuba, Universidade Estadual Paulista, Araçatuba, SP, Brasil.

Graduanda de odontologia, Departamento de Odontologia Preventiva e Social, Faculdade de Odontologia de Araçatuba, Universidade Estadual Paulista, Araçatuba, SP, Brasil.

* Pós-graduanda em Odontologia, Departamento de Odontologia Preventiva e Social, Faculdade de Odontologia de Araçatuba, Universidade Estadual Paulista, Araçatuba, SP, Brasil.

**** Professora adjunta, Departamento de Odontologia Preventiva e Social, Faculdade de Odontologia de Araçatuba, Universidade Estadual Paulista, Araçatuba, SP, Brasil.

***** Professora titular, Departamento de Odontologia Preventiva e Social, Faculdade de Odontologia de Araçatuba, Universidade Estadual Paulista, Araçatuba, SP, Brasil. 
sistema integrado de transferência do paciente dirige o usuário aos diferentes níveis de atenção sem a perda de vínculo com a unidade de saúde ${ }^{1-3}$.

Para que o sistema de saúde seja efetivo, é imprescindível o acesso equitativo aos serviços, com quantidade e qualidade satisfatórias, buscando a resolubilidade ${ }^{3-5}$. Uma das formas de fomentar a qualidade e a resolubilidade do sistema é a realização de avaliações periódicas do serviço de saúde prestado.

Diante da necessidade de promover melhoria no desempenho dos prestadores de serviço, a percepção do usuário passou a ganhar cada vez mais espaço nos planejamentos governamentais como medida de avaliação da qualidade dos cuidados em saúde end $^{6,7}$

Pesquisas envolvendo a avaliação da satisfação dos usuários fornecem subsídio a gestores e equipes de saúde, capacitando-os a desenvolver práticas mais condizentes com as demandas da população. Além disso, possibilitam complementar e equilibrar a qualidade dos serviços, proporcionando melhores resultados em termos de eficiência e eficácia do sistema ${ }^{3-6,8-12}$.

Ademais, a satisfação do usuário é uma noção psicológica que pode ser facilmente compreendida, podendo ser estudada no contexto da experiência global do usuário, em um ambiente de saúde após sua experiência com o serviço ${ }^{13}$. E ainda pode ser utilizada, não apenas como uma medida de avaliação geral da qualidade, mas também como uma medida de desempenho do serviço prestado nas unidades de saúde da família ${ }^{13}$. Quando o serviço de saúde tem bom funcionamento, o usuário responde com maior adesão ao tratamento ${ }^{4}$, com continuidade aos cuidados em longo prazo, procura por prevenção de agravos à saúde além de indicar o serviço para outras pessoas ${ }^{14}$.

Portanto, objetivou-se neste estudo avaliar a satisfação do usuário com o serviço público odontológico de acordo com suas características socioeconômicas e demográficas e com a resolubilidade do serviço prestado.

\section{Materiais e método}

Esta pesquisa foi realizada com usuários do serviço odontológico do Sistema Único de Saúde (SUS), no âmbito da Atenção Primaria à Saúde, de um município de médio porte (181.579 habitantes $)^{15}$ do estado de São Paulo.

Para calcular o tamanho amostral, realizou-se teste estatístico por meio do software Epi Info, versão 7.1.4 (Centers for Disease Control and Prevention, Atlanta, Estados Unidos), levando em conta a população adulta estimada no município investigado, a prevalência de $50 \%$ de adultos satisfeitos com a qualidade dos serviços odontológicos, efeito de desenho 1, precisão de $5 \%$ e intervalo de confiança de $95 \%$, o que resultou em 384 indivíduos. A fim de minimizar as perdas, foram acrescidos $20 \%$, totalizando 461 usuários.

A amostra foi selecionada de forma estratificada, considerando-se o universo investigado: usuários que buscaram espontaneamente o serviço público odontológico ou foram referenciados às unidades básicas de saúde e unidade de saúde da família e pelos agentes comunitários. A coleta de dados ocorreu na totalidade das unidades de saúde do município de estudo $(\mathrm{n}=12)$, em dias e períodos alternados de funcionamento, visando alcançar a multiplicidade de usuários e a representatividade da amostra.

Utilizou-se como critérios de inclusão: ser usuário do serviço odontológico da Atenção Primária à Saúde do SUS; ter idade igual ou superior a 18 anos; preferencialmente ter concluído a experiência de utilização do serviço odontológico ou estar sob tratamento odontológico. Consideraram-se critérios de exclusão: usuários menores de 18 anos; indivíduos em primeira consulta odontológica e indivíduos que estavam em consulta de urgência.

O formulário utilizado na coleta foi adaptado de dois protocolos de programas propostos pelo Ministério da Saúde brasileiro para avaliação dos serviços públicos de saúde, o Programa Nacional de Avaliação dos Serviços de Saúde) (Pnass) ${ }^{16}$ e o Programa Nacional de Melhoria do Aceso e da Qualidade da Atenção Básica (Pmaq) ${ }^{9}$. Para garantir a compreensão do instrumento, um estudo piloto foi previamente realizado, no entanto, os resultados desse estudo não foram incluídos na presente pesquisa.

O local de eleição para realização da entrevista foi a sala de espera. Todos os usuários presentes e que se enquadravam nos critérios de inclusão do estudo foram convidados a participar.

Os dados foram coletados por um único pesquisador previamente treinado para realizar a entrevista individualmente, de maneira padronizada, sem influenciar nas respostas. Foram dados esclarecimentos sobre o objetivo da pesquisa e os benefícios que ela poderia proporcionar para o melhor funcionamento do serviço de saúde e consequentemente para o próprio usuário.

Após a entrevista, os participantes receberam kits de higiene bucal e foram instrumentalizados com o auxílio de um manual informativo impresso, desenvolvido especialmente para a pesquisa, sobre temas de interesse, como saúde bucal e funcionamento do SUS, saúde e cidadania, direitos e deveres dos cidadãos.

Os dados foram digitados e processados, utilizando-se o programa estatístico Epi Info, versão 7.1.4 (Centers for Disease Control and Prevention, Atlanta, Estados Unidos). Foi realizada a análise descritiva e aplicado o teste estatístico de associação Qui-Quadrado, ao nível de significância de 5\%.

A variável dependente estudada foi satisfação do usuário, e as variáveis independentes foram: gênero, estado civil, escolaridade, idade, ocupação, 
condições socioeconômicas e resolubilidade dos problemas bucais. A resolubilidade foi avaliada com a resposta do usuário à pergunta: "Em sua opinião, o dentista desta unidade de saúde está conseguindo resolver todos os seus problemas de saúde bucal?".

A pesquisa obteve aprovação do Comitê de Ética em Pesquisa com Seres Humanos da Faculdade de Odontologia de Araçatuba, da Universidade Estadual Paulista (Parecer no $353.893 / 2013$ ), respeitando os ditames da Resolução 466/2012 do Conselho Nacional de Saúde Brasileiro.

O projeto teve apoio do Conselho Nacional de Desenvolvimento Científico e Tecnológico (CNPq) e financiamento do Ministério da Saúde - Chamada MCTI/CNPq/MS-SCTIE-Decit no 10/2012 - para Pesquisa em Saúde Bucal (Processo nº 403176/2012-3).

\section{Resulltados}

Do total entrevistado ( $\mathrm{n}=461), 449$ usuários do serviço público odontológico responderam à pergunta eleita como variável dependente do estudo, sendo considerados apenas esses sujeitos para comporem a amostra final.

A Tabela 1 mostra a relação da satisfação de usuários com o serviço público odontológico de acordo com suas condições socioeconômicas e demográficas. A maior parte da amostra foi composta por adultos jovens, do gênero feminino, com renda de até dois salários mínimos, solteiros e satisfeitos com o serviço odontológico. Quando comparadas as características da amostra à satisfação, apenas a ren$\mathrm{da}$ apresentou diferença significativa $(\mathrm{p}<0,0002)$.

Tabela 1 - Distribuição de usuários segundo condições socioeconômicas e demográficas e satisfação com o serviço público odontológico, Araçatuba, SP, 2013-2014 ( $n=449)$

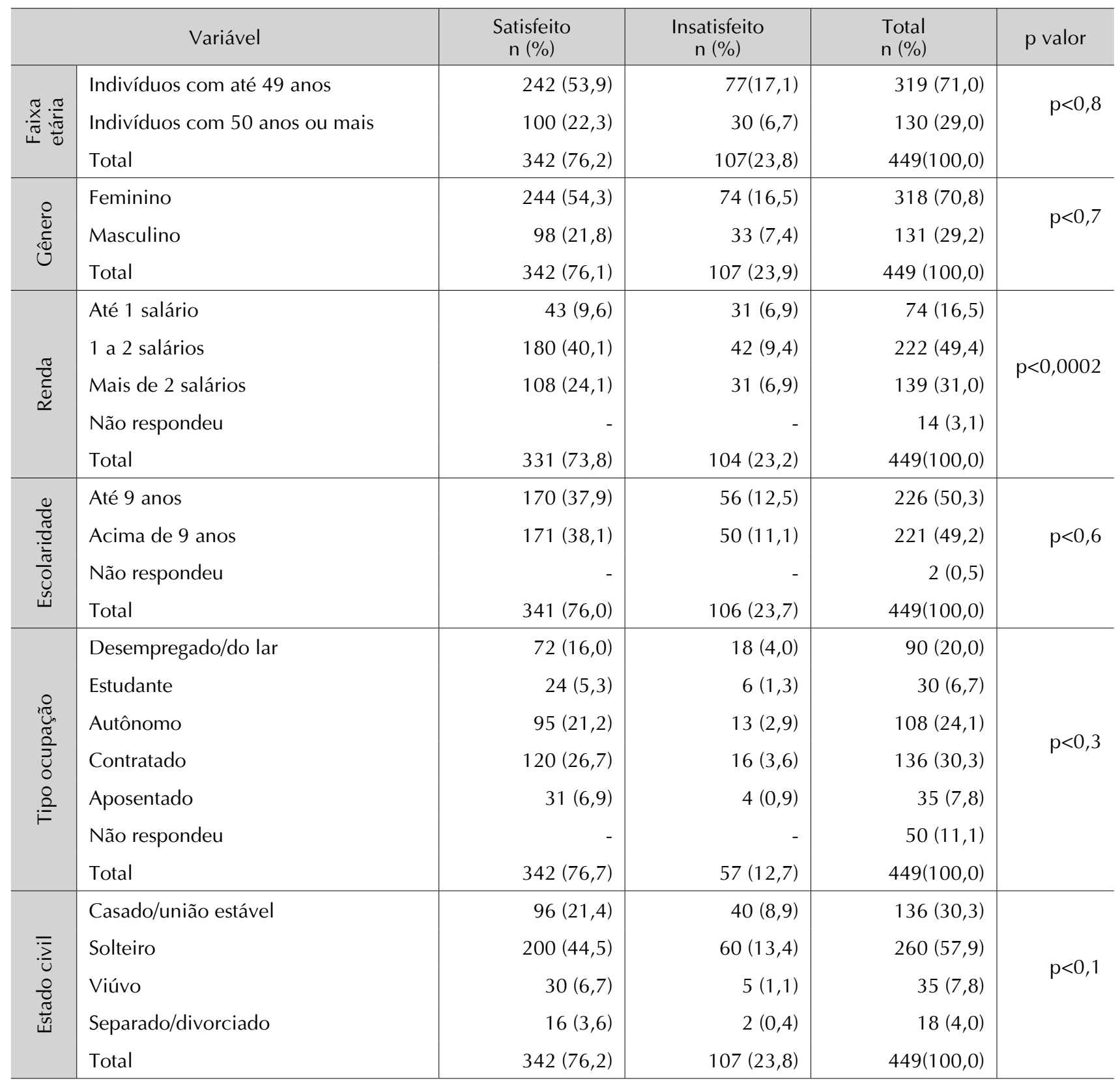

Fonte: todas as tabelas são de elaboração dos autores com base nos dados da pesquisa. 
A relação entre a resolubilidade e a satisfação com o serviço odontológico está descrita na Tabela 2. Os usuários que tiveram seus problemas resolvidos apresentaram satisfação significativamente maior $(\mathrm{p}<0,0001)$.

Tabela 2 - Distribuição de indivíduos segundo a resolubilidade e a satisfação como serviço público odontológico, Araçatuba, SP, $2013-2014$ $(n=449)$

\begin{tabular}{|c|c|c|c|c|c|}
\hline \multicolumn{2}{|r|}{ Variável } & $\begin{array}{c}\text { Satisfeito } \\
\text { n (\%) }\end{array}$ & $\begin{array}{c}\text { Insatisfeito } \\
n(\%)\end{array}$ & $\begin{array}{l}\text { Total } \\
\text { n }(\%)\end{array}$ & $\mathrm{p}$ valor \\
\hline \multirow{3}{*}{ 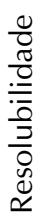 } & Resolutivo & $333(74,2)$ & $5(1,1)$ & $338(75,3)$ & \\
\hline & Não resolutivo & $9(2,0)$ & $102(22,7)$ & $111(24,7)$ & $p<0,0001$ \\
\hline & Total & $342(76,2)$ & $107(23,8)$ & $449(100,0)$ & \\
\hline
\end{tabular}

\section{Discussão}

Neste estudo, que confronta a satisfação do usuário do serviço público odontológico às suas características sociodemográficas e à resolubilidade, notou-se associação entre a renda e a solução dos problemas de saúde bucal na unidade de saúde. Os usuários com menor renda apresentaram maior satisfação com o serviço prestado. Diversas são as justificativas apontadas na literatura para elevada satisfação nesse grupo de indivíduos, como a visualização do serviço como favor ou doação e não um direito garantido por lei ${ }^{17}$, a impossibilidade de acesso ao serviço particular e a proximidade do posto de saúde à residência do usuário ${ }^{18,19}$, e a tendência em expressar menor expectativa e exigência com relação aos serviços prestados ${ }^{6,10,18-20}$.

Diferentemente do encontrado no presente estudo, Priporas et al. ${ }^{13}$ (2008), em pesquisa desenvolvida na Grécia, verificaram que usuários com menor renda apresentaram menor satisfação. $O$ fato foi justificado pelos autore ${ }^{13}$ em decorrência da desigualdade existente no tratamento de usuários com baixa renda em detrimento aos de classe social mais elevada.

A maioria dos entrevistados era de renda baixa, corroborando com outros estudos desenvolvidos com usuários do serviço público de saúde ${ }^{6,21}$. Esse fato demonstra que existe a dependência das classes mais socialmente desfavorecidas pelo serviço público de saúde ${ }^{14}$, e que a procura pelo atendimento odontológico por usuários com maior renda ocorre em consultórios e clínicas particulares ${ }^{6}$.

A resolubilidade dos serviços de saúde é uma maneira de avaliar o serviço. Quanto maior a resolubilidade de um serviço, mais voltado e preparado ele está para atender as necessidades da população ${ }^{22}$. Vieira et al. ${ }^{23}$ (2013) sugerem que o aumento da resolubilidade do serviço aconteceu a partir da criação do SUS e com a municipalização do serviço de saúde.

Os usuários que afirmaram ter todos os seus problemas de saúde bucal resolvidos pelo cirurgião-dentista da unidade de saúde apresentaram satis- fação significativamente maior. A visão do usuário é particular e os critérios que eles utilizam para a resolubilidade são desconhecidos ${ }^{23}$. Para alguns autores, a resolubilidade pode envolver aspectos relativos às tecnologias dos serviços de saúde, à formação de recursos humanos, à adesão ao tratamento e aos aspectos culturais e socioeconômicos dos usuários ${ }^{22,24,25}$. O estudo de Rosa et al. ${ }^{22}$ (2011) também associou a alta taxa de resolubilidade com os recursos instrumentais, e o conhecimento técnico do profissional à ação acolhedora e ao vínculo entre profissional e usuário.

Ainda, a literatura traz que, para alguns usuários, a resolubilidade tem associação com o atendimento especializado $0^{5,22,26,27}$, colocando no especialista a expectativa de ser cuidado e protegido ${ }^{22}$. Além disso, o usuário pode relatar também que o problema de saúde bucal foi resolvido simplesmente pelo fato de ter sido atendido na unidade de saúde, por ter sua queixa principal resolvida ou, ainda, por ter recebido algum procedimento, mesmo sem ter recebido alta.

Para garantir a atenção integral e a resolução de todos os problemas dos usuários, o serviço de saúde deve atuar em rede, de modo que o sistema referência e contrarreferência funcione adequadamente , $^{2,12,21-23,26}$. A Rede de Atenção à Saúde compreende arranjos organizativos de ações e serviços de saúde que, integradas por meio de sistemas de apoio técnico, logístico e de gestão, buscam garantir a integralidade do cuidado ${ }^{28}$.

Pesquisas mostram que quando os usuários relatam insatisfação em relação à resolubilidade, os apontamentos principais referem-se à necessidade de mais vagas para 0 atendimento cirúrgico-restaurador, à falta do tratamento reabilitador no serviço local e à falta de um esquema que referencie o usuário para a atenção especializada ${ }^{14,18,21,22,27}$.

Neste estudo, as variáveis idade, gênero, estado civil, escolaridade e ocupação não tiveram associação significativa com a satisfação, corroborando com diversos autores ${ }^{10,14,18,29}$. Contudo, para Lima et al. ${ }^{18}$ (2010), Al-Doghaither et al. ${ }^{26}$ (2001), Rahmqvist et al. ${ }^{30}(2010)$, usuários mais jovens tendem a apresen- 
tar satisfação significativamente menor com o serviço de saúde. Já para Priporas et al. ${ }^{13}$ (2008), usuários com menor escolaridade tendem a mostrarem-se mais insatisfeitos devido a falhas de comunicação com os profissionais de saúde e menor compreensão acerca do funcionamento do sistema. Outro estudo ${ }^{19}$ afirma que a população com um menor grau de escolaridade tende a emitir menos juízo de valor e ser mais condescendente com o serviço de saúde prestado, expressando graus mais elevados de satisfação.

O perfil da amostra neste estudo foi de jovens, solteiros, escolaridade baixa e que tinham uma ocupação. Para Gouveia et al. ${ }^{6}$ (2011), pessoas com melhor educação teriam melhor conhecimento sobre a sua situação de saúde e, em geral, melhores condições socioeconômicas, o que possibilitaria a procura de alternativas de atendimento, incluindo aquelas pagas, o que justificaria o maior uso do serviço público por pessoas de baixa escolaridade.

A maior utilização do serviço odontológico por adultos jovens pode ser justificada devido à grande parte da população idosa ser edêntula. Em estudo ${ }^{31}$, a população idosa relatou a baixa necessidade de tratamento odontológico devido a perdas dentárias, tendo a visão de que cuidados odontológicos são necessários apenas na presença de dentes naturais.

Ainda, o perfil dos usuários entrevistados foi predominantemente feminino, o que também foi verificado em outros estudos envolvendo usuários do

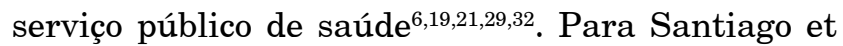
al. ${ }^{32}$ (2013), o alto número de mulheres demonstra a importância delas como usuárias do serviço público e como cuidadoras da saúde de sua família. A menor participação dos homens pode ser decorrente de um maior vínculo com o trabalho, buscando alternativas para a assistência, e também pela não priorização desses usuários nas unidades de saúde ${ }^{19,32}$.

\section{Conclusão}

Esta pesquisa permitiu verificar que a satisfação dos usuários com o serviço público odontológico foi elevada, e esteve associada à resolução dos problemas odontológicos percebidos.

\section{Abstract}

Introduction: One way of promoting the quality and resolubility of the health system is performing an assessment of health services provided, however, because of the complexity involved, this attitude is still a challenge for most services. Objective: To assess user satisfaction regarding public dental care, according to their socioeconomic and demographic characteristics, and resolubility of the service provided. Methods: It is a cross-sectional inquiry-type research, which study population were dental care users of the Unified Health System. Sample calculation was performed considering the estimated adult population, with 5\% accuracy, 95\% confidence interval, and design effect 1 , for a prevalen- ce of $50 \%$ of adults satisfied, totaling 461 users. The technique used for data collection was standardized interpersonal interview, conducted in Health Units with a structured questionnaire. Results: The sample consisted mostly of women $(70.8 \%)$, young adults $(71.0 \%)$, unmarried $(57.9 \%)$, low income $(49.4 \%)$, and low level of education (50.3\%). Most users were satisfied with the service provided (76.2\%) and satisfaction was not statistically associated with gender, age, marital status, level of education, and occupation ( $p>0.05)$, however, regarding user income and resolubility of the service, the association was significant $(p<0.05)$. Conclusion: It is concluded that the interviewed users showed high satisfaction with dental care, and income and resolubility were the only factors established as incisive for user satisfaction.

Keywords: Health Assessment. Primary Health Care. Oral Health.

\section{Referências}

1. Magalhães R, Senna MCM. Local implementation of the Family Health Program in Brazil. Cad Saúde Pública 2006; 22(12):2549-59.

2. Oliveira MS, Artmann E. Regionalização dos serviços de saúde: desafios para o caso de Angola. Cad Saúde Pública 2009; 25(4):751-60.

3. Nunes AA, Caccia-Bava MCGG, Bistafa MJ, Pereira LCR, Watanabe MC, Santos V et al. Resolubilidade da Estratégia Saúde da Família e unidades básicas de saúde tradicionais: contribuições do PET-saúde. Rev Bras Educ Med 2012; 36(1):27-32.

4. Robles ACC, Grosseman S, Bosco VL. Satisfação com o atendimento odontológico: estudo qualitativo com mães de crianças atendidas na Universidade Federal de Santa Catarina. Ciênc Saúde Coletiva 2008; 13(1):43-9.

5. Moimaz SAS, Marques JAM, Saliba O, Garbin CAS, Zina LG, Saliba NA. Satisfação e percepção do usuário do SUS sobre o serviço público de saúde. Physis 2010; 20(4):1419-40.

6. Gouveia GC, Souza WV, Luna CF, Szwarcwald CL, Souza Júnior PRB. Satisfação dos usuários com a assistência de saúde no estado de Pernambuco, Brasil, 2005. Ciênc Saúde Coletiva $2011 ; 16(3): 1849-61$.

7. Oliveira RS, Magalhães BG, Gaspar GS, Rocha RACP, Góes PSA. Avaliação do grau de satisfação dos usuários nos serviços de saúde bucal da Estratégia de Saúde da Família. Rev Bras Pesq Saúde 2009; 11(4):34-8.

8. Serapioni M, Silva MGC. Evaluation of the quality of Family Healthcare program in municipalities of Ceará: a multidimensional approach. Ciênc Saúde Coletiva 2011; 16(11):4315-26.

9. Brasil. Ministério da Saúde: Portaria no 1.654, de 19 de julho de 2011. Institui, no âmbito do Sistema Único de Saúde, o Programa Nacional de Melhoria do Acesso e da Qualidade da Atenção Básica (PMAQ-AB) e o Incentivo Financeiro do PMAQ-AB, denominado Componente de Qualidade do Piso de Atenção Básica Variável - PAB Variável. Brasília: Diário Oficial da República Federativa do Brasil; 2011. Seção 1. p. 79.

10. Bjertnaes OA, Sjetne IS, HestadIversen H. Overall patient satisfaction with hospitals: effects of patient-reported experiences and fulfilment of expectations. BMJ Qual Saf [periódico on-line] 2011 [citado 2015 Jun 17]; [telas]. Disponível em URL: http://qualitysafety.bmj.com/content/early/2011/08/26/ bmjqs-2011-000137.short. 
11. Mohammed S, Souares A, Bermejo JL, Sauerborn R, Dong $\mathrm{H}$. Performance evaluation of a health insurance in Nigeria using optimal resource use: health care providers perspectives. BMC Health Serv Res 2014; 14:127.

12. Saliba NA, Nayme JGR, Moimaz SAS, Cecilio LPP, Garbin CAS. Organization of the demand for a Centre of Dental Specialties. Rev Odontol Unesp 2013; 42(5):317-23.

13. Priporas CV, Laspa C, Kamenidou I. Patient satisfaction measurement for in-hospital services: a pilot study in Greece. J Med Mark 2008; 8(4):325-40.

14. Ricci NA, Wanderley FS, Oliveira MS, Rebelatto JR. O hospital-escola de São Carlos: análise do funcionamento por meio da satisfação dos usuários. Ciênc Saúde Coletiva 2011; 16(1):1125-34.

15. Instituto Brasileiro de Geografia e Estatística. Dados censitários de 2011 [acesso em 26 maio 2015]. Disponível em URL: http://cidades.ibge.gov.br/xtras/perfil.php?lang=\&codmun=3 50280\&search=sao-paulo I aracatuba.

16. Brasil. Ministério da Saúde. Programa Nacional de Avaliação de serviços de saúde: edição 2004-2005. Brasília: Ministério da Saúde; 2004.

17. Brasil. Lei no 8.142 , de 28 de dezembro de 1990. Dispõe sobre a participação da comunidade na gestão do Sistema Único de Saúde (SUS) e sobre as transferências intergovernamentais de recursos financeiros na área de saúde e dá outras providências. Brasília: Diário Oficial da República Federativa do Brasil; 31 dez. 1990. Seção 1, p. 25694.

18. Lima ACS, Cabral ED, Vasconcelos MMVB. Satisfação dos usuários assistidos nos Centros de Especialidades Odontológicas do Município do Recife, Pernambuco, Brasil. Cad Saúde Pública 2010; 26(5):991-1002.

19. Brandão ALRBS, Giovanella L, Campos CEA. Avaliação da atenção básica pela perspectiva dos usuários: adaptação do instrumento EUROPEP para grandes centros urbanos brasileiros. Ciênc Saúde Coletiva 2013; 18(1):103-14.

20. Bleich SN, Özaltin E, Murray CJL. How does satisfaction with the health-care system relate to patient experience? Bull World Health Organ 2009; 87:271-8.

21. Andrade KLC, Ferreira EF. Avaliação da inserção da odontologia no Programa Saúde da Família de Pompéu (MG): a satisfação do usuário. Ciênc Saúde Coletiva 2006; 11(1):123-30.

22. Rosa RB, Pelegrini AHW, Lima MADS. Resolutividade da assistência e satisfação de usuários da Estratégia Saúde da Família. Rev Gaúcha Enferm 2011; 32(2):345-51

23. Vieira V, Andrade FR, Castro CGJ, Bighetti TI, Narvai PC. Municipalização de serviços de saúde segundo profissionais de saúde bucal em um município do interior do estado de São Paulo, Brasil. Saúde Soc 2013; 22(3):795-803.

24. Paiva DCP, Bersusa AAS, Escuder MML. Avaliação da assistência ao paciente com diabetes e/ou hipertensão pelo Programa Saúde da Família do Município de Francisco Morato, São Paulo, Brasil. Cad Saúde Pública 2006; 22(2):377-85.

25. Lima MADS, Ramos DD, Rosa RB, Nauderer TM, Davis R. Acesso e acolhimento em unidades de saúde na visão dos usuários. Acta Paul Enferm 2007; 20(1):12-7.

26. Al-Doghaither AH, Abdelrhman BM, Majzoub MM. Patients' satisfaction with primary health care centers services in Kuwait city, Kuwait. J Family Community Med 2001; 8(3):59-65.

27. Borghi GN, Vazquez FL, Cortellazzi KL, Guerra LM, Bulgareli JV, Pereira AC. A avaliação do sistema de referência e contrarreferência na atenção secundária em Odontologia. RFO UPF 2013; 18(2):154-9.

28. Brasil. Ministério da Saúde. Portaria n 4.279 , de 30 de dezembro de 2010. Estabelece diretrizes para a organização da
Rede de Atenção à Saúde no âmbito do Sistema Único de Saúde (SUS). Brasília: Diário Oficial da República Federativa do Brasil; 31 dez. 2010. Seção 1. p. 88.

29. Jorge MSB, Guimarães JMX, Vieira LB, Paiva FDS, Silva DR, Pinto AGA. Avaliação da qualidade do programa Saúde da Família no Ceará: a satisfação dos usuários. Rev Baiana Saúde Pública 2007; 31(2):258-68.

30. Rahmqvist M, Bara AC. Patient characteristics and quality dimensions related to patient satisfaction. Int J Qual Health Care 2010; 22(2):86-92.

31. Peres MA, Iser BPM, Boing AF, Yokota RTC, Malta DC, Peres KG. Desigualdades no acesso e na utilização de serviços odontológicos no Brasil: análise do Sistema de Vigilância de Fatores de Risco e Proteção para Doenças Crônicas por Inquérito Telefônico (VIGITEL 2009). Cad Saúde Pública 2012; 28 (Supl. 1):S90-100.

32. Santiago RF, Mendes ACG, Miranda GMD, Duarte PO, Furtado BMASM, Souza WV. Qualidade do atendimento nas Unidades de Saúde da Família no município de Recife: a percepção dos usuários. Ciênc Saúde Coletiva. 2013; 18(1):35-44.

\section{Endereço para correspondência:}

Suzely Adas Saliba Moimaz

Rua José Bonifácio, 1193

Departamento de Odontologia Infantil e Social

16015-050 Araçatuba/SP

Telefone: (18) 3636-3249

E-mail: sasaliba@foa.unesp.br

Recebido: 23 /11/15. Aceito: 19/01/16 